www.jmscr.igmpublication.org

Impact Factor (SJIF): 6.379

Index Copernicus Value: 71.58

ISSN (e)-2347-176x ISSN (p) 2455-0450

crossref DOI: https://dx.doi.org/10.18535/jmscr/v6i5.137

Journal Of Medical Science And Clinical Research

\title{
A Comparative Study on Efficacy of Risperidone versus Risperidone and Sodium Valproate Combination in the Treatment of Aggression in Children and Adolescents with Intellectual Disability
}

\author{
Authors \\ Aparna Ramakrishnan ${ }^{1 *}$, Zara Ahmed ${ }^{2}$, Sanjiv Kale ${ }^{3}$ \\ ${ }^{1}$ Department of Psychiatry, GGMC and Sir JJ Group of Hospitals, Mumbai -08, India \\ ${ }^{2}$ Department of Psychiatry, Jamia Hamdard University, South West Delhi, India - 26 \\ Email: zaraahmed1988@gmail.com \\ ${ }^{3}$ Department of Psychiatry, D Y Patil University, Navi Mumbai, India - 400706 \\ Email: sanjivkale58@gmail.com \\ *Corresponding Author \\ Aparna Ramakrishnan \\ Contact: 9819358626. Email: dr.aparna214@gmail.com
}

\begin{abstract}
Introduction: Aggression is a symptom seen in multiple disorders. Children with Intellectual disability may exhibit aggression- harm to self, others or property. While Risperidone has been shown to produce significant reductions in aggression in youth, research has shown that mood stabilizers like Valproate can reduce aggressive symptoms effectively. Few studies are available comparing efficacy of the two drugs alone or in combination, in treatment of aggression in this population. This study compares efficacy of Risperidone versus Risperidone and Sodium valproate combination in treatment of aggression in children and adolescents with intellectual disability.

Materials and Methods: In this study,30 children and adolescents aged 4-17 years, diagnosed with Intellectual Disability (mild to moderate in severity) and aggressive behaviour (as evaluated by Modified Overt Aggression Scale) (MOAS), treated with Risperidone were compared with 30 age matched children and adolescents with Intellectual Disability and Aggression ,on a combination of Risperidone and Sodium Valproate. Follow up evaluation of the groups using MOAS was conducted at 3 weeks, 6 weeks and 6 months of treatment. Data was statistically analyzed.

Results: Children and adolescents treated with Risperidone and Sodium Valproate combination showed significantly less verbal aggression and aggression towards property. No significant differences were found in the two groups in terms of Auto aggression and Physical aggression after 6 months.

Conclusion: A combination of Sodium Valproate and Risperidone was better than Risperidone alone in decreasing verbal and aggression towards property in intellectually disabled children and adolescents.

Keywords: Aggression, Intellectual disability Risperidone Sodium Valproate.
\end{abstract}

\section{Introduction}

Aggression itself, is not a syndrome: it is a non pathognomic symptom that represents a common phenotype to multiple illnesses. Aggressive symptomatology presents across multiple psychiatric, developmental and behavioural 
disorders complicating the diagnosis and treatment of the underlying pathology. ${ }^{1}$ Verbal aggression can manifest in the form of verbal hostility, shouting/ cursing and threats of physical attack. Other forms of aggression include aggression against property, displayed as slamming doors, kicking or throwing objects, defacing walls, breaking furniture and setting things on fire. Physical injury towards oneself or auto-aggression may manifest in the form of skin picking, hair pulling, hitting or banging head or self, self mutilating behaviours or Deliberate Self Harm (DSH) attempts. Physical aggression includes violent actions such as striking, pushing, hitting or scratching others to inflict pain, bodily harm or even death.

Physical aggression in children is a major public health problem. Not only is childhood physical aggression a precursor of physical and mental health problems that will be visited on victims, but also aggressive children themselves are at a higher risk of alcohol and drug abuse, accidents, violent crimes, depression, suicide attempts, spouse abuse and neglectful and abusive parenting ${ }^{2}$. The most effective and appropriate management of aggression can occur when there is an understanding of the aetiology of the aggressive symptoms. ${ }^{1}$

According to DSM 5, Intellectual disability (intellectual development disorder) (ID) is a disorder with onset during the developmental period that includes both intellectual and adaptive functioning deficits in conceptual, social and practical domains.

The diagnostic criteria include A. Deficits in intellectual functions such as reasoning, problem solving, planning, abstraction, thinking, judgement academic learning and learning from experience confirmed by both clinical assessment and individualized standardized intelligence testing.

B. Deficits in adaptive functioning that result in failure to meet developmental and sociocultural standards for personal independence and social responsibility. Without ongoing support the adaptive deficits limit functioning in one or more activities of daily life such as communication, social participation and independent living across multiple environments such as home, school, work and community. The onset of intellectual and adaptive deficits is during the developmental period.

ID can be Mild, Moderate, Severe or Profound. It has an overall prevalence of approximately $1 \%$ in the general population and prevalence rates vary by age. Individuals with intellectual disability particularly those with more severe intellectual disability may also exhibit self injurious behaviour, aggression, disruptive behaviours including harm to others or property destruction. ${ }^{3}$ Behaviour disorders are frequent in children with intellectual disability, regardless of the underlying etiology ${ }^{4}$ Compared to children without Intellectual disability, the most prominent problem behaviours of children with ID are social proble$\mathrm{ms}$, attention problems and aggressive behaviourfound in nearly $50 \%$ of such children. ${ }^{5}$

Research literature shows that the prevalence of aggression among people with intellectual disability and autism ranges from 9-31\%. ${ }^{6}$ The aggression seen can be towards others ( hitting, biting or kicking) or manifest as destructive or disruptive behaviours (breaking windows, screaming) or towards self ( biting, self hitting, head banging). It can be planned or reactive (reaction to a situation or environment). Children with ID manifest aggressive behaviour more often than children with average intelligence, Etiology of aggression can be a number of medical neurological psychiatric or environmental factors. ${ }^{4}$ Studies show ,The risk of aggressive behaviour is more in greater degrees of intellectual disability, organic etiology, organic brain damage like Temporal Lobe Epilepsy, sensory disabilities, language difficulties, poor social skills poor social support and concomitant psychiatric disorders ${ }^{6}$

Common triggers for reactive aggressive behaviour are pain or inability to comprehend a minor event. Self injurious behaviour has been defined as a response that produces physical 
injury to the individuals own body. In children it is most commonly reported in association with an ID at rates ranging from $7-22 \%{ }^{4}$

Some studies have shown that more males than females exhibit aggression. Aggressive behaviour is exhibited more frequently or more intensely in adolescents and young adults. Aggression may also be modestly associated with certain causes of intellectual disability. Research into behavioural phenotypes has suggested that certain genetic syndromes like Downs syndrome, fragile $\mathrm{X}$ syndrome may be associated with lower rates of aggression than other forms of intellectual disability. ${ }^{6}$

The appropriate diagnosis and treatment of aggression in children and adolescents presents a unique dilemma in clinical care. ${ }^{1}$

Risperidone is a novel antipsychotic drug that has been tried in the treatment of several child psychiatric disorders. ${ }^{7}$ Risperidone is the most extensively studied medication for the treatment of aggression in youth and has been shown to produce significantly greater reductions in aggression compared with placebo. ${ }^{5}$ Risperidone or Olanzapine may decrease aggression and Self Injurious Behaviour in children with ID. ${ }^{4}$ In an open labelled study using Risperidone to treat disruptive behaviours and self injury in children with Downs syndrome, severe intellectual disability and co morbid autism spectrum disorders, it was found that children with disruptive behaviour and self injury showed the greatest improvement with Risperidone. ${ }^{8}$ The short term efficacy of Risperidone was examined in a 6 week multicentered double blind parallel group study of 118 children (aged 5-12 yrs) with severely disruptive behaviours and subaverage intelligence (IQ between 36and 84). The Risperidone group showed significantly greater improvement than placebo group in scales evaluating conduct problem, irritability, aggressive/ destructive behaviour, social withdrawal and hyperactivity. ${ }^{9}$ In a 8 week randomized double blind placebo controlled trial, it was found that subjects (aged 5-12 yrs with
Pervasive Developmental Disorder) had a significantly greater mean decrease in irritability scores (nearly double that of placebo treated subjects). ${ }^{10}$ Risperidone may be effective for severe aggression in adolescents with disruptive behaviour disorders and subaverage intelligence. ${ }^{11}$ However research has shown that mood stabilizers can also reduce aggressive symptoms and hence have become an appealing alternative in the treatment of aggression, mood lability and impulsivity in paediatric population. ${ }^{1}$ Of all Anti Epileptic Drugs (AEDs) the most evidence based data currently exists for the use of Valproate in treatment of aggression. ${ }^{1}$ In studies done by Donovan and colleagues, significant improvement was seen in disruptive adolescents who had explosive mood disorder and were given open label Valproate for 5 consecutive weeks. ${ }^{12} \mathrm{~A}$ later double blind crossover trial of youth with mood lability, explosive temper showed $86 \%$ response rate with Valproate as compared to $25 \%$ for placebo $^{13}$. Results from various studies implicate Valproate as the most efficacious AED in treatment of paediatric aggression. ${ }^{14,15}$ Case series and case reports suggest efficacy of Valproate for aggression in children and adults with mental retardation and associated co morbidities. A study examining the effects of Divalproaex sodium for irritability and aggression in children and adolescents with Autism Spectrum Disorders (ASD) over a period of 12 weeks showed statistically significant improvement in irritability in Divalproaex subjects $(62.5 \%)$ versus placebo (9\%) subjects. ${ }^{16}$

Hence the treatment of aggression in intellectually disabled children and adolescents presents a clinical conundrum in terms of the type of medication, dosage and side effects. There are few studies available comparing efficacy of Risperidone, Sodium Valproate and combination of the two in treatment of aggression in this population, especially in Indian settings, hence the need for this study. This study aims to compare efficacy of Risperidone and a combination of Risperidone and Sodium valproate in treatment of 
aggression in children and adolescents with intellectual disability.

\section{Materials and methods}

This is a longitudinal comparative study. The subjects for this study were 60 children and adolescents, aged 4-17 years (who satisfied the inclusion and exclusion criteria) diagnosed with intellectual disability (of mild to moderate severity) with aggressive behaviour (as evidenced by evaluation with Modified Overt Aggression Scale) (MOAS),. 30 subjects were treated with Risperidone alone and 30 subjects were treated with a combination of Risperidone and Sodium valproate. Both groups were matched with respect to age and sex.

Sociodemographic data of the participants was collected. A written informed consent was taken for participation from parents of the participants, who were, prior to consent, informed that refusal to participate will not alter the course of the treatment. Detailed psychiatric assessment and data regarding current psychiatric status was obtained using a predesigned proforma. Ethical clearance was sought prior to undertaking the study.

\section{Inclusion Criteria}

Children and adolescents between 4-17 years of age

Children and adolescents with mild to moderate intellectual disability (according to Kamat Binet IQ test and the DSM 5 criteria)

Participants whose parents had given a written informed consent

\section{Exclusion Criteria}

Children younger than 4 years and adolescents older than 17 years of age

Children with a history of seizure disorder or head injury

Aggressive behaviour was evaluated using MOAS (Modified Overt Aggression Scale). Evaluation was conducted at first visit and then follow up evaluations were conducted at 3weeks, 6 weeks and 6 months. Data was statistically analyzed using t test.

\section{Results}

The subjects of the study were 60 intellectually disabled, aggressive children and adolescents.

Group $1-(\mathrm{n}=30) \quad$ Subjects treated with Risperidone alone

Group $2-(n=30)$ Subjects treated with a combination of Risperidone and Sodium Vaproate

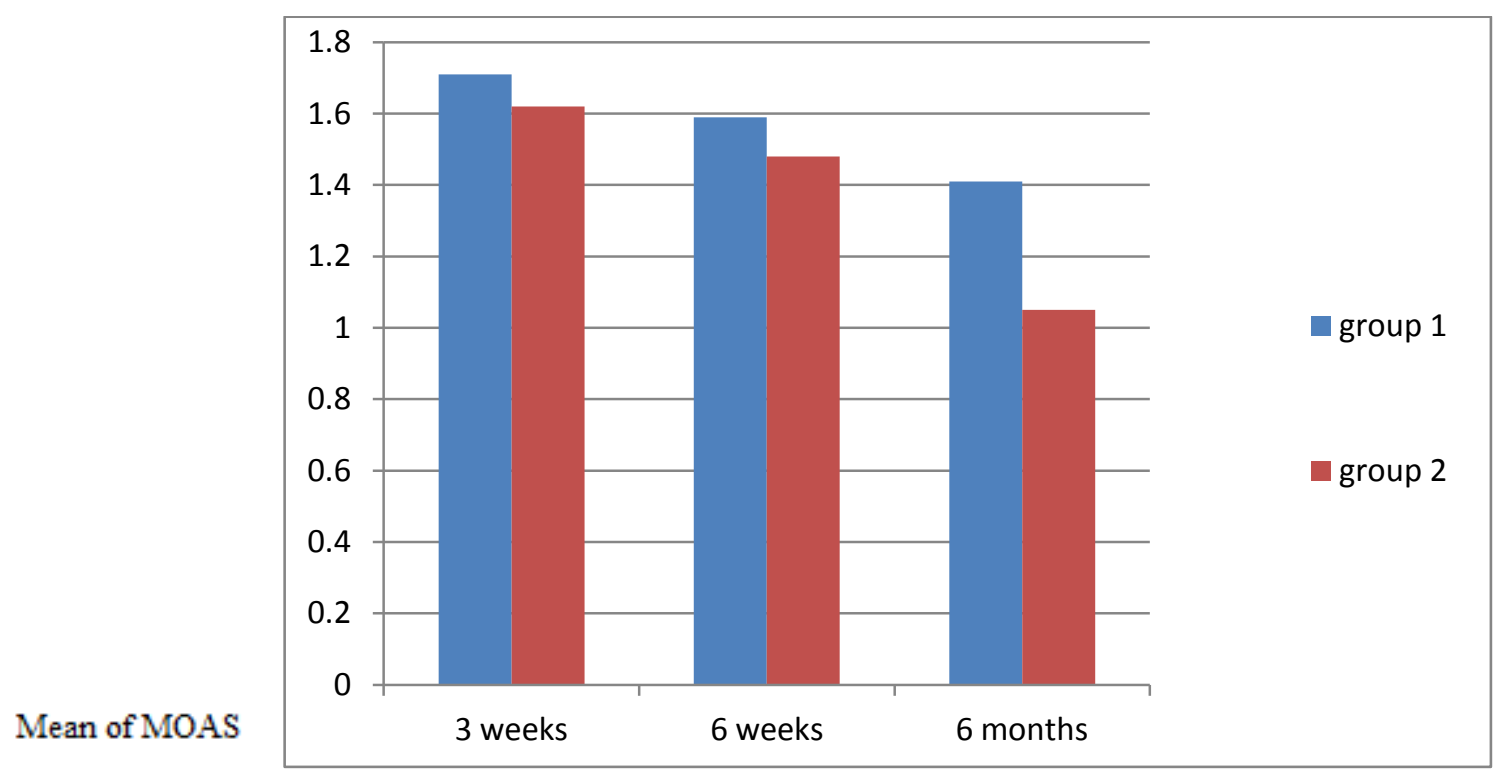

Evaluation done at

Table 1 Verbal aggression

A statistically significant difference $(\mathrm{p}=0.011)$ of verbal aggression at 6 months. Individuals in was seen between the two groups in the parameter group 2 - who were on a combination of 
Risperidone and Sodium Valproate were significantly less aggressive verbally and were less likely to shout or curse, make statements that would inflict psychological harm on others through devaluation or degradation or make threats about physical attacks.

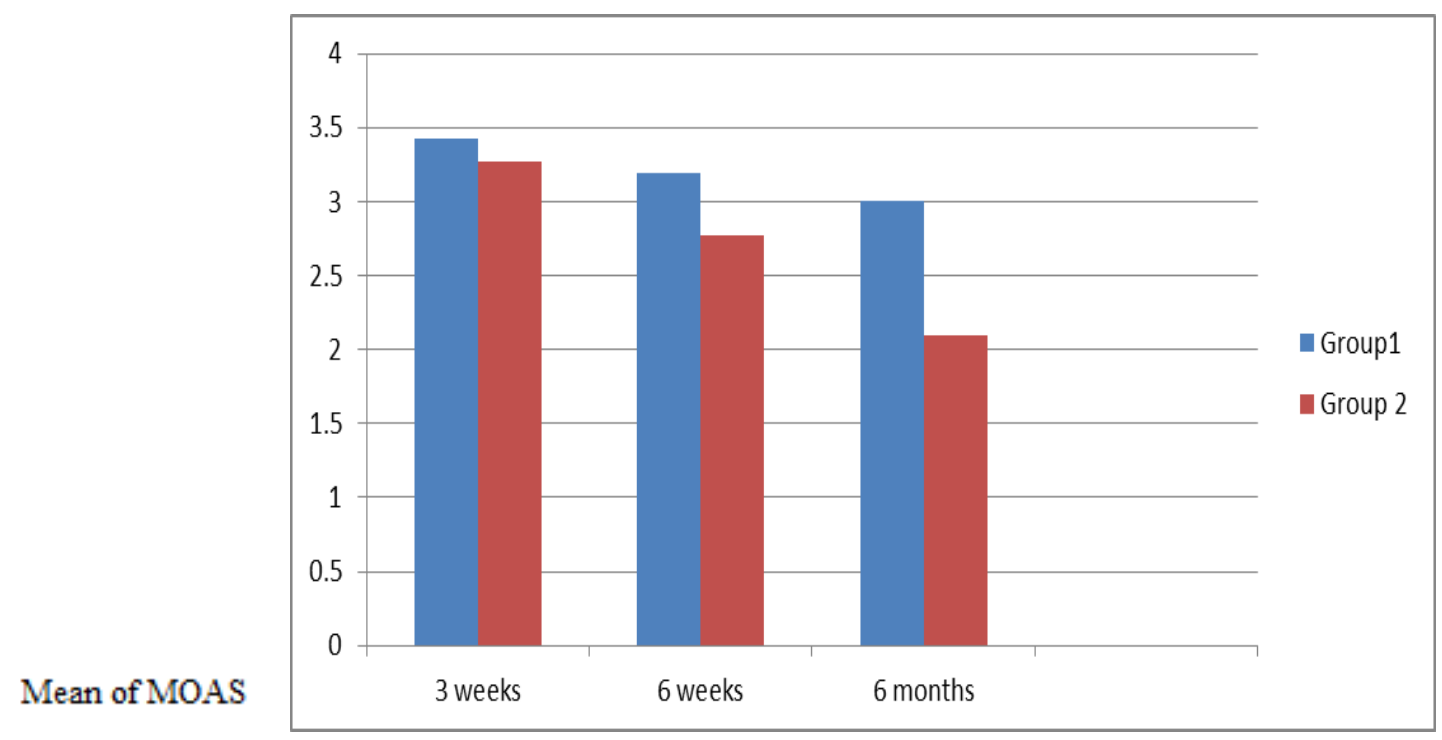

Table 2

Aggression against property

A statistically significant difference was observed $(\mathrm{p}=0.07)$ between the two groups at 6 months in this parameter.. Individuals who were on a combination of Risperidone and Sodium Valproate were found to be less engaged in wanton and reckless destruction of objects and furniture, defacing walls, smashing windows, urinating on the floor.

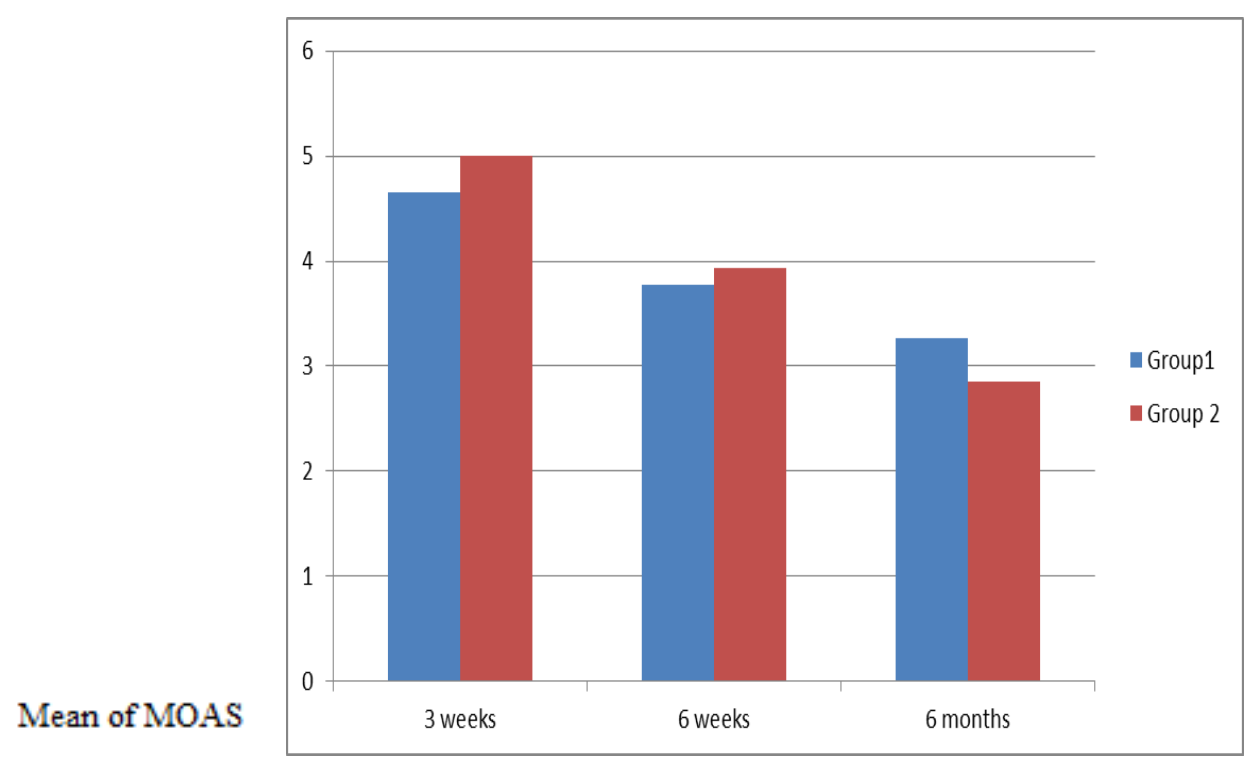

Table 3

\section{Auto aggression}

In the parameter of auto aggression which involves physical injury to self in the form of skin picking, scratching, hair pulling, hitting or cutting self and deliberate self harm, there was no

\section{Evaluation done at} significant difference in the two groups, those treated with Risperidone alone or with a combination of Risperidone and Sodium Valproate at 3 and 6 weeks. However, Improvement in this parameter was observed in both groups at 6 months of treatment. 


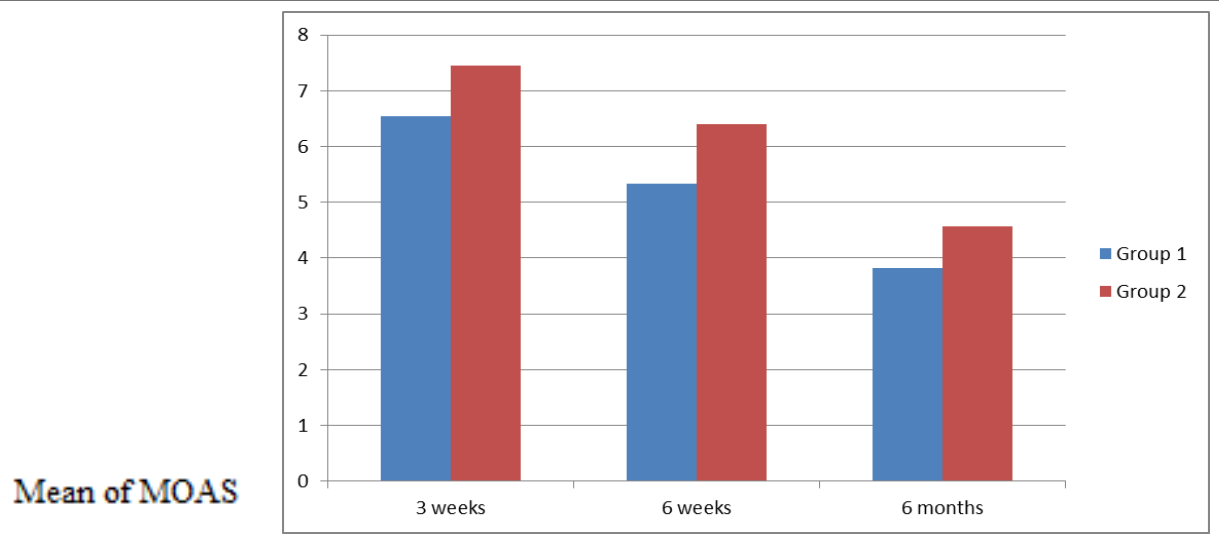

Evaluation done at

Table 4

Physical Aggression

A statistically significant difference was seen in the parameter of physical aggression between the two groups $(\mathrm{p}=0.04)$ at 3 weeks. Individuals on risperidone monotherapy were significantly less physically aggressive at 3 weeks, although this difference seemed to peter out by the end of 6 months, with both modalities of treatment showing similar outcomes.

\section{Discussion}

Behavioural disorders are a common occurrence in children with intellectual disability ${ }^{4}$ Characteristics of aggression implicate different etiologies. The underlying pathology can be inferred from the severity and duration of the aggressive episode. The most effective and appropriate management of aggression can occur when there is an understanding of the etiology of the aggressive symptoms. Focusing treatment to address a specific target of core symptoms can better guide treatment decisions. ${ }^{1}$

Despite the efficacy of atypical anti-psychotics, there are patients who do not respond to monotherapy. A possible augmentation strategy can include combined treatment with an AED. Also, Although the atypical antipsychotics are very effective and used fairly commonly in the treatment of paediatric aggression, there is concern about their use in the pediatric population due to the incidence of metabolic abnormalities, diabetes mellitus, weight gain, insulin resistance, hyperlipidemia, neuroleptic malignant syndrome, dystonias and other extra- pyramidal reactions, including possible tardive dyskinesia. Newer AEDs have improved side effect profiles and have shown some efficacy in adults for treating aggressive symptoms. AEDs may represent a more tolerable long-term treatment for aggression in children and adolescents. $^{1}$

This is a novel study comparing the efficacy of Risperidone and a combination of Risperidone and Sodium Valproate in treatment of aggression in children and adolescents with intellectual disability. On research, few comparative studies, similar to this one were available especially in the Indian Set up.

In this study the efficacy of combination of Risperidone and Sodium Valproate is significantly more than Risperidone alone in terms of verbal aggression and aggression against property in the long term (6 months). This is similar to the findings of Donovan et al. ${ }^{12,13}$ In a meta analysis done by Huband et al , Sodium valproate/divalproex was superior to placebo for outpatient men with recurrent impulsive aggression, for impulsively aggressive adults with cluster B personality disorders, and for youths with conduct disorder, but not for children and adolescents with pervasive developmental disorder. However, the authors, themselves considered that the body of evidence was insufficient to allow any firm conclusion to be drawn about the use of antiepileptic medication in the treatment of aggression and associated impulsivity. Four antiepileptics (valproate/ divalproex, carbamazepine, oxcarbazepine and phenytoin) were effective, compared to placebo, 
in reducing aggression in at least one study, although for three drugs (valproate, carbamazepine and phenytoin) at least one other study showed no statistically significant difference between treatment and control conditions.17 In this metanalysis, however, there were no comparisions of AEDs with antipsychotics or with combination of AED and antipsychotic, like in our study

There was no statistically significant difference between the two groups on the parameter of physical injury to self or auto aggression, though auto aggression was found to be reduced at 6 months with both modalities of treatment. Treatment with Risperidone alone showed a statistically significant improvement in parameter of physical aggression in the first 3 weeks.The findings are similar to Buitelaar et al who found Risperidone effective for aggression directed towards self in adolescents with below average intelligence $^{11}$ Some Studies also say, When monotherapy is not sufficient, combining an AED with either lithium or an atypical antipsychotic can result in better efficacy in aggression $^{1}$

In our study, differences in efficacy between the two groups in terms of physical aggression, petered off at 6 months. This may indicate that while short term treatment with Risperidone is more effective in management of physical aggression in intellectually disabled children, in the long run, Mood stabilizers like Valproate or AED and antipsychotic combination are equally effective.

Behaviour disorders and aggression are frequent in children with an intellectual disability and are often disabling, and can create problems in everyday life. Such behaviours are often chronic and are further complicated by the fact that parents often do not seek help for the problem, perhaps believing that it is due to the child's disability and cannot be treated. The current management of behaviour disorders and aggression in children and youth with an ID focuses on symptomatic treatment us. And few studies are available evaluating the effectiveness of various treatment options in a substantial population of such children and adolescents. From this study, It is clear that further research is required to help identify best practices for behaviour disorders in children and youth with an Intellectual Disability. Parents and professionals need to be educated about the importance of prevention and the optimal management of such disorders.

The limitations of this study are the small sample size of the study. In this study, Severe intellectually disabled individuals, individuals with borderline intelligence or Learning Disability weren't included in the study which may have influenced the results. Also, Aggression in Intellectual disability was the only factor considered. Aggression rates in different diagnoses like ASD, Downs Syndrome, Mood disorders and the differential responses for the various treatment modalities depending upon the diagnoses was not done. This is an area of further research

\section{Conclusions}

This study concludes that a combination of Sodium Valproate and Risperidone is better than Risperidone alone in decreasing verbal and aggression towards property in intellectually disabled children and adolescents over a long duration. However both modalities of treatment are equally effective in reducing physical aggression and auto aggression in this population.

\section{References}

1. Munshi KR, Oken T, Guild DJ, Trivedi H et al. The use of antiepileptic drugs for treatment of Pediatric Aggression and Mood disorders. Pharmaceuticals (Basel). 2010. Sep;3(9): 2986-3004.

2. Tremblay RE, Nagin DS,Seguin JR,Zoccolillo $\mathrm{M}$ et al. Physical aggression during early childhood : trajectories and predictors. Pediatrics. $2004 \mathrm{Jul} ; 114(1)$ : 43-50 
3. American Psychiatric association:

Diagnostic and Statistical Manual of Mental Disorders, Fifth edition. Arlington VA, American Psychiatric association, 2013: 33- 41

4. Ageranioti - Belanger $\mathrm{S}$, Brunet $\mathrm{S}$, D'Anjou G, Tellier $G$ et al. Behaviour disorders in children with an Intellectual Disability. Paediatr Child Health 2012; 17(2): 84-88

5. Dekker MC, Koot HM, Van der Ende J, Verhulst FC. Emotional and behavioural problems in children and adolescents with and without intellectual disability. Journal of Child Psychology and Psychiatry. Nov 2002.Vol 43, Iss 8: 1087-1098

6. Sturmey Peter. Treatment intervention for people with aggressive behaviour and Intellectual Disability. Autism and Related disorders. The basic handbook for Mental Health, primary care and other professionals. Editors - Geraldine Holt and Nick Bouras. 2002 produced on behalf of World Psychiatric Association by the Royal College of Psychiatrists : 42-56

7. Eapen V, Gururaj AK. Risperidone treatment in 12 children with developmental disorders and Attention deficit/hyperactivity disorder. Prim Care Companion J Clin Psychiatry. 2005;7(5):221-224

8. Capone GT, Goyal P,Grados M, Smith Bet al. Risperidone use in children with Down syndrome, severe intellectual disability and comorbid Autistic spectrum disorders : a naturalistic study. J Dev Behav Pediatr 2008:29: 106-116

9. Aman MG,De Smedt G, Derivan A, Lyons B et al. Double blind placebo controlled study of Risperidone for the treatment of disruptive behaviours in children with subaverage intelligence. Am $\mathrm{J}$ of Psychiatry 2002; 159:1337-1346
10. Shea S, Turgay A, Carrol A et al. Risperidone in the treatment of disruptive behavioural symptoms in children with Autistic and other pervasive developmental disorders.Peadiatrics 2004;114: e634-641

11. Buitelaar JK, Van der Gaag RJ,CohenKettenis P, Melman CT. A randomized controlled trial of Risperidone in the treatment of aggression in hospitalized adolescents with subaverage cognitive abilities. J. Clin Psychiatry. 2001. Apr;62(4) : 239-248

12. Donovan SJ,Susser ES, Nunes EV, Stewart JW et al.Divalpraoex treatment of disruptive adolescents : a report of 10 cases. J Clin Psychiatry .1997 ;58: 12-15

13. Donovan SJ, Stewart JW, Nunes EV, Quitkin FM et al. Divalpraoex treatment for youth with explosive temper and mood lability : a double blind, placebo controlled crossover design. Am .J. Psychiatry. 2000; $157: 818-820$

14. MacMillan CM, Korndorfer SR, Rao S, Fleisher CA et al. A comparison of Divalproaex and Oxcarbazepine in aggressive youth with Bipolar disorder. J.Psychiatr.Pract. 2006: 12 : 214-222

15. Kowatch RA, Suppes T,Carmody TJ,Bucci J et al. Effect size of Lithium, Divalproaex Sodium and Carbamzepine in children and adolescents with bipolar disorder. J.Am.Acad.Child Adolesc. Psychiatry. 2000;39:713-720

16. Hollander E, Chaplin W, Soorya L, Wasserman $\mathrm{S}$ et al. Divalproex sodium vs placebo for the treatment of irritability in children and adolescents with Autism Spectrum Disorders. Neuropsychopharmacology. 2010; 35: 990-998

17. Huband N, Ferriter N, Nathan R, Jones H. Cochrane database system rev. 2010 Feb17; (2) 